\title{
Bilateral Salpingectomy with Oophorectomy
}

National Cancer Institute

\section{Source}

National Cancer Institute. Bilateral Salpingectomy with Oophorectomy. NCI Thesaurus.

Code $\mathrm{C51765.}$

Surgery to remove both fallopian tubes, as well as both ovaries. 